\title{
EFECTO DEL SOMBRÍO SOBRE LA SIGATOKA NEGRA (Mycosphaerella fijienșis MORELET) EN CULTIVO DE PLÁTANO cv HARTÓN (MUSa AAB SIMMONDS)
}

\section{SHADOW EFFECT ON BLACK SIGATOKA (Mycosphaerella fijiensis MORELET) IN PLANTAIN CULTIVATION cv HARTON (Musa AAB SIMMONDS)}

\begin{abstract}
José Barrera V. ${ }^{1}$, Fernando Barraza A. ${ }^{2}$, Rodrigo Campo A. ${ }^{3}$
${ }^{1}$ Ingeniero agrónomo, M.Sc. Fisiología vegetal, Profesor investigador, Facultad de Ciencias Agrícolas, Departamento de Ingeniería Agronómica y Desarrollo Rural, Grupo de investigación Agricultura Sostenible. Universidad de Córdoba, carrera 6 No. 76-103, Montería, Córdoba, Colombia, e-mail: jlbarrera@correo.unicordoba.edu.co; ${ }^{2}$ Ingeniero agrónomo, M.Sc. Horticultura, Ph.D. Horticultura, Profesor investigador, Facultad de Ciencias Agrícolas, Departamento de Ingeniería Agronómica y Desarrollo Rural, Grupo de investigación Agricultura Sostenible. Universidad de Córdoba, e-mail: fbarraza@correo.unicordoba. edu.co; ${ }^{3}$ Ingeniero agrónomo, M.Sc. Protección de Cultivos, Ph.D. Fitopatología, Profesor investigador, Facultad de Ciencias Agrícolas, Departamento de Ingeniería Agronómica y Desarrollo Rural, Grupo de investigación Agricultura Sostenible. Universidad de Córdoba, e-mail: rocampoarana@correo.unicordoba.edu.co
\end{abstract}

Rev. U.D.C.A Act. \& Div. Cient. 19(2): 317-323, Julio-Diciembre, 2016

\section{RESUMEN}

La sigatoka negra en el cultivo del plátano es considerada como una de las enfermedades más limitante de la producción, a nivel mundial, siendo manejada con fungicidas, ocasionando contaminaciones ambientales e incremento de los costos de producción. Como estrategia no contaminante, algunos investigadores recomiendan cultivar en condiciones de sombrío, para reducir la enfermedad. El objetivo de este trabajo fue evaluar el efecto de diferentes porcentajes de sombrío en la incidencia de la enfermedad y en la producción, bajo condiciones del Caribe húmedo colombiano. En condiciones de campo, se estableció el cultivar 'Hartón', en un diseño de bloques completos al azar y cuatro porcentajes de sombrío, 20, 35 y 50\%, teniendo como testigo plantas cultivadas a libre exposición solar $100 \%$. Las evaluaciones, se realizaron durante el periodo de floración del cultivo, cuantificando el número de hojas funcionales NHF, la hoja más joven con síntomas HMJS, la hoja más joven enferma HMJE, el índice de severidad IS y rendimiento. Los tratamientos con 20,35 y $50 \%$ de sombrío no presentaron diferencias estadísticas entre sí; más sí, con el testigo en las variables NHF, HMJE, IS y rendimiento. Los tratamientos con sombra manifestaron menor IS de la sigatoka negra, de 13 al 15\%, mientras el testigo fue del 18\%; además, los rendimientos se incrementaron en $400 \mathrm{~kg} \mathrm{ha}^{-1}$. Se concluye, que el cultivo de plátano 'Hartón', manejado a partir del 20\% de sombrío, fue suficiente para reducir la severidad de la sigatoka negra e incrementar significativamente los rendimientos.

Palabras clave: Epidemiología, fisiología, manejo de sigatoka negra.

\section{SUMMARY}

Black sigatoka in plantain cultivation is considered one of the most limiting production diseases worldwide. It is managed with fungicides, causing environmental pollution and increased production costs. As a nonpolluting strategy, some researchers recommend cultivating shade conditions to reduce the disease. The aim of this study was to evaluate the effect of different shade percentages in the incidence of disease and production under the Caribbean Colombian humid conditions. Under field conditions it was established to cultivate the 'Harton' in a completely randomized block design and four shade percentages 20,35 and $50 \%$, having as control plants growing in $100 \%$ sun exposure. Evaluations were performed during the flowering period of the crop, quantifying the number of functional leaves NFL, the youngest leaf with symptoms YLS, the youngest diseased leaf YDL, the severity index SI and yield. Treatments with 20, 35 and 50\% shade were not statistically different from each other; but with the 
witness in the NFL, YLS, SI and yield. Treatments with shade had lower SI of black sigatoka, between 13 to $15 \%$; while the witness was $18 \%$; also the yield increased in $400 \mathrm{~kg} \mathrm{ha}^{-1}$. It is concluded that growing 'Harton', managed from $20 \%$ shade was enough to reduce the severity of black sigatoka and significantly increase yields.

Key words: Epidemiology, physiology, management of black sigatoka.

\section{INTRODUCCIÓN}

La enfermedad más limitante de la producción mundial de plátanos durante los últimos cincuenta años, con cualquier cultivar, ha sido la sigatoka negra (Hidalgo et al. 2006). Es causada por el hongo Mycosphaerella fijiensis Morelet, caracterizándose por la alta producción de ascosporas y conidias asexuales de su anamorfo Paracercospora fijiensis, produciendo un alto número de ciclos sexuales y asexuales por año y colonización rápida de los tejidos foliares de cultivos de plátano y de banano, causando necrosis parcial o total, reduciendo la fotosíntesis y ocasionando madurez prematura de los frutos, con disminución hasta de un $50 \%$ del rendimiento, dependiendo de la severidad del ataque (Rodríguez \& Cayón, 2008; De Bellaire et al. 2010).

Para la reducción de la sigatoka negra, se recomienda un manejo temprano, mediante la aplicación de estrategias, que incluyen deshojes, despunte de hojas y aplicación de fungicidas. Este último rubro constituye el $27 \%$ de los costos de producción del cultivo y se estima que al año se realizan entre 30 y 50 aplicaciones de fungicidas, tanto protectantes como sistémicos, con consecuencias negativas como fitotoxicidad, pérdida de sensibilidad de $M$. fijiensis a diversos grupos químicos, contaminación ambiental y presencia de residuos tóxicos en los frutos (Etebu \& Young, 2011).

En el proceso de llenado de los frutos de la planta de plátano, el desarrollo progresivo del área foliar y su actividad fisiológica son factores determinantes en el desempeño productivo, por lo cual, es fundamental que durante su crecimiento y desarrollo, la planta tenga suficientes hojas funcionales, que garanticen que la fotosíntesis se lleve a cabo, de manera óptima (Arcila et al. 2000). En este sentido, para obtener una buena producción de racimos de plátanos, se deben mantener, al momento de la floración, un mínimo ocho hojas funcionales (Torres \& Hernández, 2004). Lo anterior, indica la importancia del manejo agronómico para llegar a la floración, por lo menos, con ocho sanas libres de sigatoka negra (Barrera et al. 2009).

El cultivo de plátano, como planta $\mathrm{C}_{3}$, requiere de baja luminosidad entre 30 a $80 \%$, para obtener los mejores rendimientos (Siles et al. 2013); no obstante, en Colombia, se siembran 45.500 ha a libre exposición, como monocultivo (ICA, 2014). Al interior del país, se establece en asocio con especies vegetales que le producen sombra, siendo común encontrar cultivos de café o cacao, asociados con plátano frutales y forestales (ICA, 2014; Siles et al. 2013).

Teniendo en cuenta que el manejo de la Sigatoka negra con fungicidas causa contaminación ambiental e incrementos en los costos de producción, en este trabajo, se planeó investigar, bajo las condiciones de la zona productora de Tierralta, Córdoba, el manejo de la Sigatoka negra, con una alternativa no contaminante, como es el uso de diferentes niveles de sombrío y su influencia en los rendimientos.

\section{MATERIALES Y MÉTODOS}

La investigación, se llevó a cabo entre el 11 de marzo 2012 y el 10 de febrero de 2013, en el municipio de Tierralta Córdoba, ubicado geográficamente a $08^{\circ} 10$ '34" latitud Norte y $76^{\circ} 03^{\prime} 46^{\prime \prime}$ longitud Oeste, con temperatura anual promedio de $27,3^{\circ} \mathrm{C}$, humedad relativa de $82 \%, 1144 \mathrm{~mm}$ de precipitación anual y altitud de 51msnm.

Bajo condiciones de campo, se establecieron plantas de dos meses de edad, del cultivar Hartón, en un diseño de bloques completos al azar, con tres repeticiones y cuatro porcentajes de sombrío: 20, 35 y 50\%, teniendo como testigo, plantas cultivadas a libre exposición $100 \%$. Cada repetición estuvo conformada por una parcela con 18 plantas, distanciadas a 2,7m en triángulo. La separación entre parcelas fue de 3,0m y la superficie total del experimento fue de $1518 \mathrm{~m}^{2}$, en donde estuvieron ubicadas un total de 216 plantas.

Para establecer el sombrío, se utilizaron mallas de polisombra, color negro, ubicadas a 5,0m de altura, calibradas para dejar pasar el 80,65 y $50 \%$ de la radiación solar, sujetadas mediante estacas de mangle y alambre, calibre número 10. Los porcentajes de radiación incidente en las parcelas fueron verificados mediante lecturas con un fotómetro-radiómetro cuántico Li-Cor ${ }^{\circledR} 184$.

Evaluación de la sigatoka negra. La severidad, se evaluó seis meses después del establecimiento de los diferentes niveles de sombra, cuando las plantas iniciaban la floración; durante dos meses, cada quince días, se evaluaron tres plantas en estado de floración, en cada tratamiento, empleando la escala propuesta por Stover, modificada por Gauhl (Orjeda 1998). Los datos de severidad fueron transformados a Índice de Severidad IS, mediante la siguiente ecuación, propuesta por Towsend y Heuberguer (Orjeda 1998):

$$
\mathrm{IS}=\sum \mathrm{nb} /(\mathrm{N}-1) \mathrm{T} * 100
$$

donde: 
IS = Índice de severidad

$\mathrm{n}=$ Número de hojas en cada nivel

$\mathrm{b}=$ Grado de severidad

$\mathrm{N}=$ Número de grado máximo en la escala $=7$

$\mathrm{T}=$ Número total de hojas evaluadas

Otras variables, medidas por tratamiento, fueron el número de hojas funcionales, hoja más joven con síntomas y rendimiento.

El número de hojas funcionales NHF fueron aquellas que, visualmente, presentaban el $80 \%$ de su superficie libre de necrosis por sigatoka negra y se cuantificaron, enumerándolas de arriba hacia abajo, a partir de la hoja bandera, de acuerdo con la metodología propuesta por Hernández et al. (2005).

La hoja más joven con síntomas HMJS correspondió a la primera hoja totalmente abierta, contada de arriba hacia abajo, que presentó, por lo menos, 10 manchas de lesiones discretas necrosadas de sigatoka negra, con lesiones deprimidas y centro de color claro. La hoja más joven enferma HMJE correspondió a aquella contada de arriba hacia abajo, que presentó estrías fácilmente visibles desde el suelo (Torrado Jaime \& Castaño Zapata, 2008).

Los racimos no fueron embolsados ni se les hizo desmanes. El rendimiento, se determinó mediante la cosecha de las cuatro plantas centrales de cada parcela, a los 75 días después de la emisión de la inflorescencia. Finalmente, los racimos se pesaron y se estimaron los rendimientos en $t \cdot \mathrm{ha}^{-1}$.

Los datos obtenidos de las variables NHF, HMJS, HMJE, IS y rendimiento, para cada tratamiento, fueron sometidos a análisis de varianza, previa revisión del cumplimiento de la normalidad de los datos; posteriormente, se realizaron comparaciones de las medias Tukey $\mathrm{p} \leq 0,05$, empleando el programa estadístico SAS 9.0.

\section{RESULTADOS Y DISCUSIÓN}

Numero de hojas funcionales NHF. Hubo diferencias estadísticas significativas entre los tratamientos, donde los tratamientos con sombrío fueron significativamente diferentes con el testigo, en todas las fechas evaluadas (Tabla 1).

Tabla 1. Número de hojas funcionales NHF del cultivo de plátano Hartón, durante el periodo de floración, en diferentes porcentajes de sombrío.

\begin{tabular}{|c|c|c|c|c|c|}
\hline \multirow{2}{*}{$\begin{array}{c}\text { Sombrío } \\
\mathbf{\%}\end{array}$} & \multicolumn{5}{|c|}{ NHF } \\
\cline { 2 - 6 } & $\mathbf{2 6 0}$ días* & $\mathbf{2 7 5}$ días & $\mathbf{2 9 0}$ días & $\mathbf{3 0 5}$ días & $\mathbf{3 2 0}$ días \\
\hline 20 & $10,6 \mathrm{a}$ & $10,6 \mathrm{a}$ & $11,3 \mathrm{a}$ & $11,4 \mathrm{a}$ & $12,0 \mathrm{a}$ \\
\hline 35 & $11,2 \mathrm{a}$ & $11,4 \mathrm{a}$ & $11,3 \mathrm{a}$ & $11,5 \mathrm{a}$ & $12,0 \mathrm{a}$ \\
\hline 50 & $10,5 \mathrm{a}$ & $11,0 \mathrm{a}$ & $11,1 \mathrm{a}$ & $11,5 \mathrm{a}$ & $11,0 \mathrm{a}$ \\
\hline 0 & $8,5 \mathrm{~b}$ & $8,3 \mathrm{~b}$ & $9,5 \mathrm{~b}$ & $7,6 \mathrm{~b}$ & $7,2 \mathrm{~b}$ \\
\hline
\end{tabular}

*Días después de plantado; Promedios con letras distintas, dentro de una misma columna, indican diferencia estadística según la prueba de Tukey $(\mathrm{p} \leq 0,05)$.

La menor cantidad de hojas funcionales, se encontró en el tratamiento a libre exposición, indicando que los diferentes porcentajes de sombrío proporcionaron al cultivo un efecto positivo sobre el número de hojas funcionales sanas. Estos resultados coinciden con las investigaciones de Martínez (2003), quien reporta una mayor sanidad, determinada con un mayor NHF, cuando el cultivo es efectuado en ambiente de $50 \%$ de sombrío; igualmente, Belalcázar et al. (1991) indican que es necesario el establecimiento de un sistema agroforestal simultáneo con el cultivo de plátano a distancias, que no interfieran directamente con las plantas, como por ejemplo, $4 \mathrm{~m}$ entre hileras de plantas y $2 \mathrm{~m}$ entre plantas en la hilera, puesto que ayuda a disminuir la severidad de la enfermedad de la sigatoka negra.
En estudios realizados por Barrera et al. (2009), concluyeron que plantas de plátano que conservaron entre ocho y doce hojas durante la floración presentaron mayor tamaño de racimos. Estos mismos autores indican que para garantizar un buen llenado es necesario que la planta a cosecha termine, al menos, con seis hojas funcionales. Por otra parte, Cayón (2001) y Siles et al. (2013) reportan que las mayores tasas de fotosíntesis ocurren en las hojas más jóvenes de la planta dos, tres, cuatro y cinco, reduciéndose, drásticamente, en las hojas seis, siete, ocho y nueve, más viejas, lo que indica, la posibilidad que al cultivo le favorece el sombrío en etapas tempranas del crecimiento, para su vigor general y mejor respuesta al ataque de plagas y de enfermedades. 
Hoja más joven con síntoma HMJS. El análisis de varianza presentó diferencias estadísticas significativas entre los tratamientos, donde los tratamientos con sombrío fueron concluyentemente diferentes con el testigo, en todas las fechas evaluadas, presentando un mayor número de hojas sanas (Tabla 2). Entre los diferentes niveles de sombrío, no hubo diferencias significativas de HMJS, mas sí de éstos con el testigo, presentándose resultados similares a los obtenidos en cultivos de forestales asociados con plátano, donde el primero, ejerce un efecto hasta del $50 \%$ de sombra (Karani, 1986). Las plantas de plátano a libre exposición solar fueron más sensibles a la sigatoka negra, al presentar la HMJS entre cuatro y seis (Tabla 2), las cuales, están por debajo del número de hojas necesarias para desarrollar un buen racimo, lo que va a afectar el rendimiento y la calidad de la fruta (Martínez, 2003; Almodóvar \& Díaz, 2007).
Por otra parte, Aguirre et al. (2012) indican que el cultivo bajo sombra puede reducir la presión de $M$. fijiensis, ya que se retarda, apreciablemente, el ciclo de la enfermedad y se obtienen menos hojas infectadas, con pocos impactos negativos sobre la productividad, cuando la radiación solar se reduce hasta el $20 \%$.

Hoja más joven enferma HMJE. Se presentaron diferencias estadísticas entre tratamientos. Los tratamientos con sombrío fueron estadísticamente iguales entre sí y significativamente superiores al testigo (Tabla 3). Los resultados obtenidos concuerdan con la investigación de Martínez (2003), en cuanto a que, bajo condiciones de cultivo con sombrío, en etapa de floración, la HMJE estuvo entre la número diez y once, indicando que la planta de plátano puede llegar hasta el final de su ciclo productivo, lo que le permitió un buen llenado de los frutos y, por tanto, alto rendimiento.

Tabla 2. Hojas más joven con síntomas HMJS de sigatoka negra (M. fijiensis), en cultivo de plátano Hartón, durante el periodo de floración.

\begin{tabular}{|c|c|c|c|c|c|}
\hline \multirow{2}{*}{$\begin{array}{c}\text { Sombrío } \\
\mathbf{\%}\end{array}$} & \multicolumn{5}{|c|}{ HMJS } \\
\cline { 2 - 6 } & $\mathbf{2 6 0}$ días* & $\mathbf{2 7 5}$ días & $\mathbf{2 9 0}$ días & $\mathbf{3 0 5}$ días & $\mathbf{3 2 0}$ días \\
\hline 20 & $7,2 \mathrm{a}$ & $7,3 \mathrm{a}$ & $7,2 \mathrm{a}$ & $8,1 \mathrm{a}$ & $8,7 \mathrm{a}$ \\
\hline 35 & $7,4 \mathrm{a}$ & $7,2 \mathrm{a}$ & $7,1 \mathrm{a}$ & $8,3 \mathrm{a}$ & $9,2 \mathrm{a}$ \\
\hline 50 & $6,5 \mathrm{~b}$ & $6,4 \mathrm{~b}$ & $7,3 \mathrm{a}$ & $8,2 \mathrm{a}$ & $8,4 \mathrm{a}$ \\
\hline 0 & $4,6 \mathrm{c}$ & $5,1 \mathrm{c}$ & $5,4 \mathrm{~b}$ & $5,5 \mathrm{~b}$ & $5,8 \mathrm{~b}$ \\
\hline
\end{tabular}

*Días después de plantado; Promedios con letras distintas, dentro de una misma columna, indican diferencia estadística según la prueba de Tukey $(\mathrm{p} \leq 0,05)$.

Tabla 3. Hojas más joven enferma HMJE con sigatoka negra (M. fijiensis), en cultivo de plátano Hartón, durante el periodo de floración.

\begin{tabular}{|c|c|c|c|c|c|}
\hline \multirow{2}{*}{$\begin{array}{c}\text { Sombrío } \\
\%\end{array}$} & \multicolumn{5}{|c|}{ HMJE } \\
\hline & 260 días* & 275 días & 290 días & 305 días & 320 días \\
\hline 20 & $9,3 \mathrm{a}$ & $9,7 \mathrm{a}$ & $9,8 \mathrm{a}$ & $9,7 \mathrm{a}$ & $11,0 \mathrm{a}$ \\
\hline 35 & $9,5 \mathrm{a}$ & $9,8 \mathrm{a}$ & $10,2 \mathrm{a}$ & $9,7 \mathrm{a}$ & $11,0 \mathrm{a}$ \\
\hline 50 & $9,4 \mathrm{a}$ & $9,1 \mathrm{a}$ & $9,8 \mathrm{a}$ & $10,0 \mathrm{a}$ & $10,1 \mathrm{a}$ \\
\hline 0 & $7,1 \mathrm{~b}$ & $7,5 \mathrm{~b}$ & $7,2 \mathrm{~b}$ & $8,7 \mathrm{a}$ & $9,7 \mathrm{a}$ \\
\hline
\end{tabular}

*Días después de plantado; Promedios con letras distintas, dentro de una misma columna, indican diferencia estadística según la prueba de Tukey $(\mathrm{p} \leq 0,05)$.

Para el caso de las plantas sometidas a plena exposición solar, el hecho de presentar hoja más joven enferma entre las primeras hojas de la planta, agrava la obtención de buenos rendimientos, puesto que dentro del manejo agronómico de la sigatoka negra, si se opta por realizar las operaciones de deshoje y despunte para disminuir el ascenso más rápido de la enfermedad hacia las hojas superiores, se deben cortar todas las hojas viejas e infectadas antes de que la planta comience floración, con lo que quedaría con un número de hojas por debajo de seis, que es la cantidad mínima para no afectar el rendimiento (Etebu \& Young, 2011). 
Índice de severidad IS. Hubo diferencias significativas entre tratamientos. El tratamiento con plena exposición solar superó a los tratamientos con sombrío y tuvo su mayor mag- nitud a los 290 días después de plantado (Tabla 4), lo que coincidió con lo reportado por Armario et al. (2007), para el clon FHIA 20.

Tabla 4. Índice de severidad de la sigatoka negra (M. fijiensis), en cultivo de plátano Hartón, durante el periodo de floración, en diferentes porcentajes de sombrío.

\begin{tabular}{|c|c|c|c|c|c|}
\hline \multirow{2}{*}{$\begin{array}{c}\text { Sombrío } \\
\%\end{array}$} & \multicolumn{5}{|c|}{ Índice de severidad (\%) } \\
\cline { 2 - 5 } & $\mathbf{2 6 0}$ días* & $\mathbf{2 7 5}$ días & $\mathbf{2 9 0}$ días & $\mathbf{3 0 5}$ días & $\mathbf{3 2 0}$ días \\
\hline 20 & $22,7 \mathrm{a}$ & $17,0 \mathrm{a}$ & $18,0 \mathrm{a}$ & $17,6 \mathrm{a}$ & $15,0 \mathrm{a}$ \\
\hline 35 & $22,7 \mathrm{a}$ & $16,7 \mathrm{a}$ & $18,0 \mathrm{a}$ & $17,0 \mathrm{a}$ & $13,0 \mathrm{a}$ \\
\hline 50 & $21,0 \mathrm{a}$ & $23,3 \mathrm{a}$ & $18,0 \mathrm{a}$ & $18,0 \mathrm{a}$ & $15,0 \mathrm{a}$ \\
\hline 0 & $24,0 \mathrm{a}$ & $25,0 \mathrm{~b}$ & $28,0 \mathrm{~b}$ & $19,0 \mathrm{~b}$ & $17,7 \mathrm{~b}$ \\
\hline
\end{tabular}

* Días después de plantado; Promedios con letras distintas, dentro de una misma columna, indican diferencia estadística según la prueba de Tukey $(\mathrm{p} \leq 0,05)$.

En términos generales, para todos los tratamientos, el IS presentó un comportamiento decreciente durante el periodo de floración evaluado, indicando menor magnitud para los tratamientos con sombra; posiblemente, se relacione con el aumento del área foliar de las plantas, que causa modificación de las condiciones ambientales, por el sombreamiento y la disminución de la temperatura, lo que está relacionado con la reducción de la severidad de M. fijiensis (Mobambo et al. 1993; Norgrove, 2003; Hernández et al. 2005). También está reportado que al reducir la cantidad de luz incidente en el interior de las plantaciones de plátano, se disminuye la toxicidad de cercosporin, que es una toxina de carácter fotosensible, producida por $M$. fijiensis, en el proceso de patogenicidad (Orozco et al. 2008).
Algunos autores, como Pocasangre et al. (2000) y Meneses et al. (2003), reportan que en cultivos de Musa spp., los efectos positivos de las condiciones de cultivo bajo sombra no solo están dirigidos a disminuir los daños causados por sigatoka negra, sino que, también, logran aminorar los daños causados por nematodos y por enfermedades causadas por hongos, presentes en el suelo, como Fusarium sp.

Rendimiento. Los tratamientos con los diferentes porcentajes de sombrío resultaron estadísticamente iguales entre sí y significativamente superiores al tratamiento de libre exposición de radiación solar (Tabla 5); posiblemente, se debió a la disminución del índice de severidad de $M$. fijiensis en los diferentes tratamientos con sombra, produciendo un mayor

Tabla 5. Rendimiento del cultivo de plátano Hartón, en diferentes porcentajes de sombrío.

\begin{tabular}{|c|c|}
\hline $\begin{array}{c}\text { Sombrío } \\
\%\end{array}$ & Rendimiento $\left(\mathbf{t} \cdot \mathbf{h a} \mathbf{~}^{-\mathbf{1}}\right)$ \\
\hline 20 & $9,0 \mathrm{a}$ \\
\hline 35 & $9,1 \mathrm{a}$ \\
\hline 50 & $9,3 \mathrm{a}$ \\
\hline 0 & $8,6 \mathrm{~b}$ \\
\hline
\end{tabular}

Promedios con letras distintas, dentro de una misma columna, indican diferencia estadística según la prueba de Tukey $(\mathrm{p} \leq 0,05)$.

número de hojas funcionales, mayor número de hojas sanas. Además, los diferentes porcentajes de sombrío afectaron el ambiente, en cuanto a disminución de la temperatura y aumento de la humedad relativa, siendo la planta más eficiente en los procesos de fotosíntesis, respiración y transporte de fotoasimilados, lo cual, afecta la economía del carbono del patógeno y aumenta la relación fuente-vertedero de la planta, que favorece el llenado de racimos (Eckstein et al. 1995; Israeli et al. 1995; Hidalgo et al. 2006; Refaie et al. 2012). 
Además de lo anterior y de acuerdo con Marín et al. (2003) y Nava \& Vera (2004), en las hojas enfermas por M. fijiensis, se afectan los procesos de fotosíntesis y de respiración y ocurren daños morfológicos y fisiológicos irreparables, que impiden el funcionamiento adecuado de la planta y que inciden directamente sobre su desarrollo y su producción, lo que trae como consecuencia, de acuerdo con Rodríguez \& Cayón (2008), baja productividad, maduración prematura de frutos y disminución de la calidad.

Agradecimientos. A todos los miembros del grupo de investigación en agricultura sostenible, quienes contribuyeron en la toma y en la tabulación de datos, en especial, a los Ingenieros Agrónomos Iván Pastrana, Ender Correa y Orlando Marín. Conflicto de intereses: El manuscrito fue preparado y revisado con la participación de todos los autores, quienes declaramos que no existe conflicto de intereses que ponga en riesgo la validez de los resultados presentados. Financiación: Este estudio fue financiado por la Universidad de Córdoba.

\section{BIBLIOGRAFÍA}

1. AGUIRRE, S.; PIRANEQUE, N.; MENJIVAR, J. 2012. Relación entre las propiedades edafoclimáticas y la incidencia de sigatoka negra (Mycosphaerella fijiensis Morelet) en la zona bananera del Magdalena-Colombia. RIIA (Colombia). 3(2):13-25.

2. ALMODÓVAR, W.; DÍAZ, M. 2007. Identificación y manejo de sigatoka negra y otras enfermedades de plátano y guineo. Ed. Colegio de Ciencias Agrícolas, Universidad de Puerto Rico. 30p.

3. ARCILA, M.; GIRALDO, G.; DUARTE, J. 2000. Influencia de las condiciones ambientales sobre las propiedades físicas y químicas durante la maduración del fruto de plátano Dominico-Hartón (Musa AAB Simonds) en la zona cafetera central. En: Cayón, G. (ed.). Poscosecha y agroindustria del plátano en el eje cafetero de Colombia. Ed. Universidad del Quindío (Colombia). p.101-124.

4. ARMARIO, D.; HERRERA, L.; HERNÁNDEZ, M.; ESPINOSA, A.; DÁVILA, A.; RAMÍREZ, T.; ARREDONDO, I.; TRIANA, O. 2007. Magnitud del Índice de severidad de la enfermedad sigatoka negra (Mycosphaerella fijiensis Morelet) en el cultivar S-INIVIT-1 en las condiciones edafo-climáticas del municipio Santo Domingo en Cuba. Agricultura urbana y suburbana de Cuba. 31(3):144-148.

5. BARRERA, J.; CAYÓN, G.; ROBLES, J. 2009. Influencia de la exposición de las hojas y el epicarpio de frutos sobre el desarrollo y la calidad del racimo de plátano "Hartón" (Musa AAB Simmonds). Agr. Col. 27(1):7379.

6. BELALCÁZAR, S.; MERCHÁN, V.; MAYORGA, M. 1991. Control de enfermedades. En: Belalcázar, S. (ed). El cultivo del plátano en el trópico. Ed. Feriva (Colombia). p.241-297.

7. CAYÓN, G. 2001. Evolución de la fotosíntesis, transpiración y clorofila durante el desarrollo de la hoja de plátano (Musa AAB Simmonds). INFOMUSA (Francia). 10(1):12-15.

8. DE BELLAIRE, L.; FOURÉ, E.; ABADIE, C.; CARLIER, J. 2010. Black leaf Streak disease is challenging the banana industry Fruits. 65(6): 327-342.

9. ECKSTEIN, K.; ROBINSON, J.; DAVIE, J. 1995. Physiological responses of banana (Musa AAA, Cavendish sub-group) in the subtropics. III. Gas exchanges, growth analysis and source-sink interaction over a complete crop cycle. J. Hort. Sci. (Reino Unido). 70(1):169-180.

10. ETEBU, E.; YOUNG, W. 2011. Control of black sigatoka disease: challenges and prospects. Afr. J. Agr. Res. 6(3):508-514.

11. HERNÁNDEZ, L.; HIDALGO, W.; LINARES, B.; HERNÁNDEZ, J.; ROMERO, N.; FERNÁNDEZ, S. 2005. Estudio preliminar de vigilancia y pronóstico para sigatoka negra (Mycosphaerella fijiensis Morelet) en el cultivo de plátano (Musa AAB cv Hartón) en Macagua-Jurimiquire, estado Yaracuy. Rev. Fac. Agron. 22(4):325-329.

12. HIDALGO, M.; TAPIA, A.; RODRIGUEZ, W.; SERRANO, E. 2006. Efecto de la sigatoka Negra (Mycosphaerella fijiensis) sobre la fotosíntesis y transpiracion foliar del banano (Musa sp. AAA, cv. Valery). Agron. Costarricense. 30(1):35-41.

13. INSTITUTO COLOMBIANO AGROPECUARIO -ICA-. 2014. Sanidad vegetal. El ICA proyecta vigilar $35 \mathrm{mil}$ hectáreas de plátano y banano durante este 2014 . Disponible desde internet en: http://www.ica.gov.co/ Noticias/Agricola/2013-\%281\%29/El-ICA-proyectavigilar-35-mil-hectareas-de-platan.aspx (con acceso 03/03/2016).

14. ISRAELI, Y.; PLAUT, Z.; SCHWARTZ, A. 1995. Effect of shade on banana morphology, growth and production. Sci. Hort. (Australia). 62:45-56. 
15. KARANI, P. 1986. Observations on the productivity of matoke (bananas) (Musa spp.) under agroforestry at Entebbe, Uganda. Commonw. For. Rev. 65(3):241250.

16. MARÍN, D.; ROMERO, R.; GUZMÁN, M.; SUTTON, T. 2003. Black sigatoka: an increasing threat to banana cultivation. Plant Dis. 87(3):208-222.

17. MARTÍNEZ, A. 2003. El cultivo del plátano en los Llanos orientales. Ed. Siglo XX (Colombia). 60p.

18. MENESES, A.; POCASANGRE, L.; SOMARRIBA, E.; RIVEROS, A.; ROSALES, F. 2003. Densidad de hongos endofíticos y abundancia de nematodos en plantaciones de banano y plátano de la parte baja de los territorios indígenas de Talamanca. Agrofor. Américas. (Costa Rica). 10(37-38):59-62.

19. MOBAMBO, K.; GAUHL, F.; VUYLSTEKE, D.; ORTIZ, R.; PASBERG, C.; SWENNEN, R. 1993. Yield loss in plantain from black sigatoka leaf spot and field performance of resistant hybrids. Field Crops Res. 35(1):35-42.

20. NAVA, C.; VERA, J. 2004. Relación del número de hojas a floración y hojas perdidas en el ciclo reproductivo con el peso del racimo en plantas de plátano en presencia de sigatoka negra. Rev. Fac. Agron. (LUZ). 21:335-342.

21. NORGROVE, L. 2003. Agrisilviculture systems: timberfood crop systems in the humid tropics. J. Interdiscipl. Cross-Cultural Studies. 3:1-15.

22. ORJEDA, G. 1998. Evaluación de la resistencia de los bananos a las enfermedades de sigatoka y marchitamiento por Fusarium. Ed. Louis-Jean, Gap. (Francia). 61p.
23. OROZCO, M.; OROZCO, J.; PÉREZ, O.; MANZO, G.; FARÍAS, J.; DA SILVA, W. 2008. Prácticas culturales para el manejo de la Sigatoka negra en bananos y plátanos. Trop. Plant Pathol. 33(3):189-196.

24. POCASANGRE, L.; SIKORA, R.; VILICH, V.; SCHUSTER, R. 2000. Survey on banana endophytic fungi from Central America and screening for biological control of the burrowing nematode (Rhadopholus similis). Infomusa. 9:3-5.

25. REFAIE, K.; ESMAIL, A.; MEDANY, M. 2012. The response of banana production and fruit quality to shading nets. J. Applied Sci. Res. 8(12):5758-5764.

26. RODRÍGUEZ, P.; CAYÓN, G. 2008. Efecto de Mycosphaerella fijiensis sobre la fisiología de la hoja de banano. Agr. Col. 26(2):256-265.

27. SILES, P.; BUSTAMANTE, O.; VALDIVIA, E.; BURKHARDT, J.; STAVER, C. 2013. Photosynthetic performance of banana (Gross Michel, AAA) under a natural shade gradient. Acta Hort. 986:71-77.

28. TORRADO-JAIME, M.; CASTAÑO-ZAPATA, J. 2008. Incidencia y severidad de las sigatokas negra (Mycosphaerella fijiensis Morelet) y amarilla (Mycosphaerella musicola Leach et Mulder) del plátano según los estados fenológicos. Agr. Col. 26(3):435-442.

29. TORRES, N.; HERNÁNDEZ, J. 2004. Efecto del número de hojas en el desarrollo del racimo de plátano Hartón Musa AAB. Agroalimentación \& Desarrollo Sostenible. 5(3):17-22.

Recibido: Abril 1 de 2016

Aceptado: Octubre 21 de 2016

\section{Cómo citar:}

Barrera V., J.; Barraza A., F.; Campo A., R. 2016. Efecto del sombrío sobre la sigatoka negra (Mycosphaerella fijiensis Morelet) en cultivo de plátano cv Hartón (Musa AAB Simmonds). Rev. U.D.C.A Act. \& Div. Cient. 19(2): 317-323. 